\title{
View-Volume Network for Semantic Scene Completion from a Single Depth Image
}

\author{
Yuxiao Guo ${ }^{1,2}$, Xin Tong ${ }^{2}$ \\ ${ }^{1}$ University of Electronic Science and Technology of China \\ ${ }^{2}$ Microsoft Research Asia \\ yuxiao.guo@outlook.com, xtong@microsoft.com
}

\begin{abstract}
We introduce a View-Volume convolutional neural network (VVNet) for inferring the occupancy and semantic labels of a volumetric 3D scene from a single depth image. The VVNet concatenates a 2D view CNN and a 3D volume CNN with a differentiable projection layer. Given a single RGBD image, our method extracts the detailed geometric features from the input depth image with a $2 \mathrm{D}$ view $\mathrm{CNN}$ and then projects the features into a $3 \mathrm{D}$ volume according to the input depth map via a projection layer. After that, we learn the 3D context information of the scene with a 3D volume $\mathrm{CNN}$ for computing the result volumetric occupancy and semantic labels. With combined 2D and 3D representations, the VVNet efficiently reduces the computational cost, enables feature extraction from multi-channel high resolution inputs, and thus significantly improves the result accuracy. We validate our method and demonstrate its efficiency and effectiveness on both synthetic SUNCG and real NYU dataset.
\end{abstract}

\section{Introduction}

Reconstructing and understanding a 3D scene from its partial observations is an important technique in many robotic and vision tasks, such as indoor navigation, object retrieval, and visual reasoning. Given a single depth image captured from a 3D scene, a set of methods recently have been developed [Song et al., 2017; Yang et al., 2017] for automatically predicting the semantic labels or completing 3D shapes of the objects in the scene using the convolutional neural networks (CNN). To achieve good performance, previous studies have revealed that both local geometric details and global 3D context of the scene need be learned in this task [Song et al., 2017]. The former one helps the system to identify the small objects in the scene, and the later one is used for inferring occluded objects from the scene layout. However, designing a CNN that can efficiently learn both features is a non-trivial task.

2D CNN based methods [Gupta et al., 2014; 2015] take the depth input as an additional channel of RGB image and apply 2D CNNs for scene segmentation and object detection.
Although these methods can fully exploit the high resolution input to generate detailed segmentation results, they ignore the $3 \mathrm{D}$ context information of the scene and thus cannot infer the invisible part of the scene. 3D CNN based methods [Wu et al., 2015; Nguyen et al., 2016; Song and Xiao, 2016; Yang et al., 2017] convert input depth maps or point clouds into a volumetric representation and design 3D CNNs for 3D scene segmentation or object completion. However, the high computational and memory cost of the 3D CNN limits their capability for recovering object details. Recently, Song et al.[2017] proposed SSCNet for semantic scene completion, where the system simultaneously predicts the object shapes and semantic labels from a single depth image. However, the 3D CNN used in their solution limits the input resolution and the depth of the neural networks, which leads to wrong labels and missing shape details in the results.

In this paper, we propose a cascaded convolutional neural network, named View-Volume Net (VVNet), for semantic scene completion from a single depth image. The key idea of our method is to handle the local geometric details and global 3D context with two convolutional neural networks: a $2 \mathrm{D}$ view $\mathrm{CNN}$ for extracting $2 \mathrm{D}$ geometric features, and a $3 \mathrm{D}$ volume $\mathrm{CNN}$ for learning 3D context of the scene. Given a single depth image captured from a 3D scene, our method first extracts a set of 2D feature maps from the input depth image with the $2 \mathrm{D}$ view $\mathrm{CNN}$ and then projects the feature maps into a 3D feature volume according to the input depth map via a projection layer. After that, the $3 \mathrm{D}$ volume $\mathrm{CNN}$ is applied to the $3 \mathrm{D}$ feature volume to learn the $3 \mathrm{D}$ context information and predicts the occupancy and semantic label of each voxel in the view frustum. Since the feature projection is differentiable, the cascaded VVNet can be trained end-toend.

The VVNet efficiently learns both geometry features and $3 \mathrm{D}$ context from the training dataset. The $2 \mathrm{D}$ view $\mathrm{CNN}$ avoids the high computational cost and memory consumption of the 3D CNN, which not only enables us to extract geometric features from the high-resolution input depth map, but also allows us to exploit multiple signals computed from the input depth image for feature extraction. Meanwhile, the 3D volume CNN defined over the low resolution feature volume efficiently learns the global 3D context of the scene. Moreover, the VVNet provides a flexible framework for combining variant $2 \mathrm{D}$ and $3 \mathrm{D}$ CNNs. To this end, we design and eval- 
uate a set of VVNet models with different configurations for semantic scene completion.

We train the VVNets on both synthetic SUNCG dataset and real NYU dataset and validate our design. We also compare their performance with previous methods. Experimental results demonstrate that our method outperforms the stateof-the-art methods on both datasets, with much better accuracy and three times speed up for training, as well as more than 7 times speed up for inference. With different configurations, we further offer VVNet models with different trade-offs between the training/inference cost and result accuracy. All these VVNet models achieve better accuracy than previous solutions.

\section{Related Work}

In this section, we discuss related work and focus on methods for analyzing and completing a 3D scene from depth images or 3D point clouds, as well as the deep learning approaches that are based on hybrid 2D and 3D representations. Please refer to [Ioannidou et al., 2017] for a survey of deep learning techniques for $3 \mathrm{D}$ data processing.

\subsection{D Scene Analysis}

A set of methods have been proposed for scene segmentation, scene completion, and object detection from an input RGBD image or depth image. 2D image-based methods regard the depth as an additional channel of the 2D RGB image and leverage manually-crafted features [Gupta et al., 2013; Atapour-Abarghouei and Breckon, 2017] or 2D deep neural networks [Gupta et al., 2014; 2015] for these scene analysis tasks. 3D volume-based approaches convert the input depth map into a volumetric representation and exploit manually crafted 3D features [Ren and Sudderth, 2016] or 3D CNNs [Song and Xiao, 2016] for detecting 3D objects from the input RGBD image. Although these methods can successfully detect and segment visible 3D objects and scenes in the input RGBD images, they cannot infer the scenes that are totally occluded. Instead, our method predicts semantic labeling and $3 \mathrm{D}$ shapes for both visible and invisible objects in a 3D scene.

Liu et al. [2017] introduced 3DCNN-DQN-RNN for parsing 3D point cloud of a scene. PointNet [Qi et al., 2016] and PointNet++ [Qi et al., 2017] develop deep learning framework on 3D point cloud for scene semantic labeling and other $3 \mathrm{D}$ shape analysis tasks. These methods take the $3 \mathrm{D}$ point cloud of whole 3D scene as the input. On the contrary, our method takes a single depth image for semantic scene completion.

\subsection{D Scene Completion}

Firman et al. [2016] inferred the occluded 3D object shapes from a single depth image via random forest. Zheng et al. [2013] completed the occluded scene in the input depth image with a set of pre-defined rules and refined the completion results by physical reasoning. These methods perform scene segmentation and completion in two separate steps. Recently, Song et al. [2017] proposed 3D SSCNet for simultaneously predicting the semantic labels and volumetric occupancy of the $3 \mathrm{D}$ objects from a single depth image. Although this method unifies segmentation and completion and significantly improves the result, the expensive 3D CNN limits the input volume resolution and network depth, and thus restrains its performance. By combining 2D CNN and 3D CNN, our method efficiently reduces the training and inference cost, enhances the network depth and thus significantly improves the result accuracy.

\subsection{D Object Completion}

A set of methods reconstruct the $3 \mathrm{D}$ object shape from a single depth image using 3D shape retrieval [Rock et al., 2015], Convolutional Deep Belief Network (CDBN) [Wu et al., 2015; Nguyen et al., 2016], or a 3D Generative Adversarial Networks (GAN) [Yang et al., 2017]. All these methods model the input depth maps and resulting 3D shapes with a $3 \mathrm{D}$ volumetric representation. Although these methods can be combined with other scene segmentation methods for predicting 3D shapes of the visible object in the input depth map, they cannot be used for inferring objects that are totally occluded. Our method is designed for recovering complete 3D shapes of both visible and occluded 3D objects from a single depth image of a 3D scene.

\subsection{Hybrid Representation for 3D Deep Learning}

A set of methods [Choy et al., 2016; Girdhar et al., 2016; Jimenez Rezende et al., 2016; Tulsiani et al., 2017; Yan et al., 2016; Häne et al., 2017] cascade 2D encoder and 3D decoder for reconstructing 3D object shapes from a single-view color image. Because the feature vector extracted from the $2 \mathrm{D}$ color image has no 3D position information, it is mapped to a low resolution $3 \mathrm{D}$ volume $\left(2^{3}\right.$ or $\left.4^{3}\right)$ via fully connected (FC) neural networks. In our method, the 2D features extracted from the input depth map inherit the 3D positions of the input depth map and thus can be directly mapped to a 3D volume via projection. Wang et al. [2017] combined 3D GAN and 2D recurrent convolutional networks (RCN) for reconstructing high resolution 3D shapes from corrupted 3D models, where the 2D RCN takes 2D slices of the 3D volume generated by the 3D GAN as input for completing 3D shapes. Our method takes 2D depth map as the input and applies 3D volume $\mathrm{CNN}$ to the projected $2 \mathrm{D}$ features for $3 \mathrm{D}$ scene completion. Kalogerakis et al. [2017] fused semantic segmentation results generated by $2 \mathrm{D}$ fully convolutional networks (FCN) from different views into a conditional random field defined on 3D object surface for object segmentation. Instead of directly obtaining the segmentation results in each view, the 2D View CNN in VVNet only extracts intermediate geometric features for the $3 \mathrm{D}$ Volume $\mathrm{CNN}$ to learn the $3 \mathrm{D}$ context information in the whole volume for scene completion.

\section{View-Volume Network}

Given a single-view depth image $I_{d}$ captured from a $3 \mathrm{D}$ scene, our VVNet outputs semantic label $C=c_{0}, \ldots c_{N+1}$ for each voxel of the scene inside the view frustum. Here we follow [Song et al., 2017] to denote the number of object classes as $N$ and mark all empty voxels by $c_{0}$. As illustrated in Fig. 1(b), the VVNet consists of three parts: a 2D view network for extracting 2D features from the input depth image, 


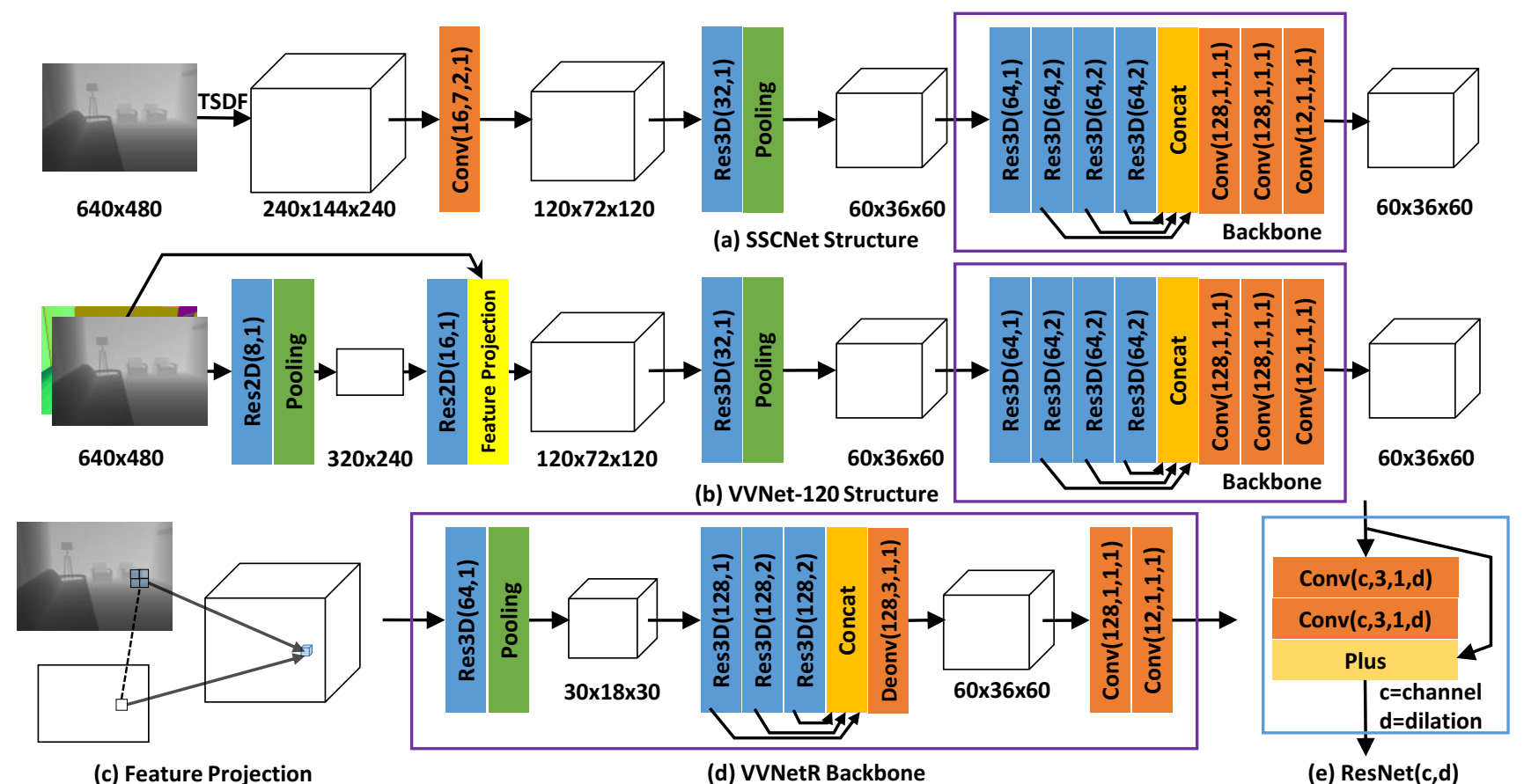

(c) Feature Projection

(d) VVNetR Backbone

(e) $\operatorname{Res} \operatorname{Net}(c, d)$

Figure 1: The network structures of VVNet and SSCNet for semantic scene completion.

a feature projection layer for converting the 2D features maps into a 3D feature volume, and a 3D volume network for inferring voxel labels from the projected feature volume. In the following part of this section, we discuss the design of each part and VVNet training.

\subsection{D View Network}

The $2 \mathrm{D}$ view network extracts $2 \mathrm{D}$ geometry features from the input depth map. For this purpose, our method first computes the normal map from the input depth image and then feeds both normal and depth maps to the 2D view network. As illustrated in Fig. 1(b), we apply the residual neural network (ResNet) structure in our 2D view network design. Each ResNet block includes two convolution layers and a shortcut from input to output as shown in Figure 1(e). When a pooling layer is applied after a ResNet block, the feature map resolution is halved and the number of feature maps is doubled. For 2D ResNet used in the view network, a batch normalization layer is applied after each convolution layer. The total number of the layers in the $2 \mathrm{D}$ view network is determined by the resolution of the target feature maps $(320 \times 240$ in Figure 1).

\subsection{Feature Projection}

As shown in Fig. 1(c), the projection layer projects the 2D feature maps constructed by the $2 \mathrm{D}$ view network into a $3 \mathrm{D}$ feature volume. The voxel size of the feature volume is set to be twice of the average distance of the neighboring depth pixels. Because the feature volume resolution is always lower than the feature map resolution, several neighboring features will be projected into the same voxel. This is equivalent to perform a pooling operation during the projection.

To project the $2 \mathrm{D}$ feature maps into the feature volume, we first construct an axis-aligned volume in the viewing coordi- nate system as in [Song et al., 2017] and then upsample the feature maps to input depth resolution with the nearest neighboring sampling. After that, we project the feature vectors of all depth pixels into the voxels of the 3D feature volume according to their viewing directions and depth values. Finally, we get the feature vector in each voxel by averaging the feature vectors projected into it. For the voxels that are not occupied by any depth image pixels, their feature vectors are zero. We found that other pooling operations (e.g. max pooling) can also be used for computing the feature vector in each voxel but have the similar affect to the result. Instead of downsampling the depth image to the feature map resolution, we apply this super-sampling scheme for feature projection so that we can avoid the holes caused by the perspective projection and large variations in the depth image. During training, we record the mapping between feature map pixels and voxels in a table for gradient back-propagation.

In our current implementation, we set the resolutions of our feature volumes to be same as the ones in the SSCNet [Song et al., 2017] for a fair comparison. We set the resolution of the feature map that corresponds to the largest 3D volume $(240 \times 144 \times 240)$ to be $640 \times 480$ because for the TSDF in this resoluton, the one constructed from half-resolution depth image $(320 \times 240)$ is almost same as the one constructed from $640 \times 480$ depth image. For the downsampled feature volumes, we scale down the resolutions of the feature maps accordingly.

\subsection{D Volume Network}

After the feature projection layer, the view-dependent 2D feature maps extracted by the view CNN are converted to a viewindependent 3D feature volume. In this step, we extract the $3 \mathrm{D}$ context of the scene and infer the semantic label of voxels 


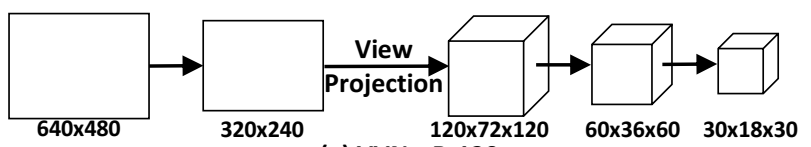

(a) VVNetR-120

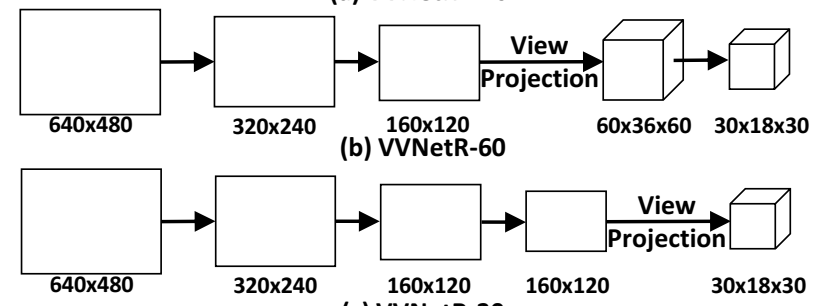

(c) VVNetR-30

Figure 2: Different trade-offs between view and volume networks in VVNetR design.

from the $3 \mathrm{D}$ feature volume via a $3 \mathrm{D}$ volume $\mathrm{CNN}$. To this end, we follow the SSCNet in our 3D feature volume CNN design(Figure 1(b)), where the 3D features are first extracted by the ResNet layers and then fed into a backbone network to generate the semantic labels for all voxels. We also design a new backbone network with enlarged reception field. As shown in Figure 1(d), the new backbone adds a new pooling layer to downsample the extracted 3D features and then applies the original backbone network to 3D features at a lower resolution. After that, we deconvolute the concatenated features to the output resolution and generate volumetric semantic labels using two convolution layers. Note that in 3D volume network, we do not apply batch normalization after each convolution layer. We denote the VVNet with the SSCNet backbone as VVNet, and the one with the new backbone as VVNetR. In Section 4, we demonstrate that our VVNetR not only reduces the computational cost for learning the $3 \mathrm{D}$ context, but also improves the result accuracy.

\subsection{Trade-off between View and Volume Networks}

The VVNet provides a flexible and general framework for combining 2D and 3D CNN for 3D scene analysis. By choosing different resolutions of the result feature maps of $2 \mathrm{D}$ view network, we can make different trade-offs between the depth of $2 \mathrm{D}$ view network and 3D volume network. Fig. 2 illustrates three VVNets, which are named as VVNetR-120, VVNetR60, and VVNetR-30, where the numbers in the name indicate the resolution of the projected feature volume. On one side, as the resolution of the projected feature volume decreases, more layers in the $3 \mathrm{D}$ volume $\mathrm{CNN}$ are replaced by the corresponding $2 \mathrm{D}$ view network layers, which results in less computations and smaller memory footprints in both network training and inference. On the other side, as more 3D volume network layers are replaced by the $2 \mathrm{D}$ view network layers, more detailed 3D context information in the scene may be lost in the projected 3D feature volume and thus leads to degradation of the result accuracy. We evaluate this trade-off in details in the next section.

\subsection{Network Training}

Given the training data set (i.e the depth images and ground truth volumetric object labels of 3D scene), the VVNet can be trained end-to-end. For this purpose, we use the voxel-wise softmax as the loss function as in [Song et al., 2017] in the network training. To compute the loss function, we remove all empty voxels in the visible free space, outside field of view and outside the room but include all non-empty voxels and occluded empty voxels. We do not apply the data balancing scheme in [Song et al., 2017] in our training process.

\section{Evaluation}

We have implemented VVNet in TensorFlow under Ubuntu 16.04 on a workstation with an Intel $6700 \mathrm{~K}$ CPU and two NVIDIA GTX 1080Ti GPUs. We use SGD optimization in VVNet training, where we set the momentum as 0.9, learning rate as 0.01 , and weight decay as 0.0005 . We found that the original SSCNet is not fully trained. For a fair comparison, we ported SSCNet in TensorFlow and trained it with more iterations ( $150 \mathrm{~K}$ iteration with batch size 4$)$. We denote our SSCNet implementation as SSCNet* in the following discussions.

Datasets We validate our method on the synthetic SUNCG [Song et al., 2017] dataset and the real NYU [Silberman et al., 2012] dataset. The SUNCG dataset consists of about $45 \mathrm{~K}$ synthetic scenes. We select the same training/test dataset used in [Song et al., 2017] for our network training and evaluation. Specifically, the training dataset includes nearly $150 \mathrm{~K}$ depth images and corresponding ground truth volume, sampled from a $8 \mathrm{~K}$ subset of the scenes. The test dataset is sampled from 170 scenes and consists of totally 470 pairs of depth image and ground truth volume. For SUNCG, we train VVNet with $150 \mathrm{~K}$ iterations and change the learning rate to 0.001 after $100 \mathrm{~K}$ iterations. We evaluate the results every 2000 steps after $130 \mathrm{~K}$ iterations, and average them as the final results.

The real NYU dataset includes 1449 depth images (795 for training, 654 for test), captured by the Kinect depth sensor. The ground truth completion and segmentation notations are from [Guo et al., 2015]. Because some manually labeled volumes and their corresponding depth images are not well aligned in the NYU dataset, we also use NYUCAD dataset in [Firman et al., 2016] in our experiments, in which the depth map is rendered from the label volume. For both NYU and NYUCAD datasets, we fine tune the VVNet models trained from SUNCG dataset with $4 \mathrm{~K}$ iterations. After that, we test the models at every 200 iterations and pick the best one as the final result.

Error Metric For each neural network, we measure the precision, recall, and IOU of all test results as in [Song et al., 2017]. The IOU measures the overlapped ratio between intersection and union of the positive prediction volume and the ground truth volume. For scene completion (SC) task, the ground truth volume includes all the occluded voxels in the view frustum. For semantic scene completion (SSC) task, the ground truth volume includes both occluded voxels and visible surface voxels.

\subsection{Ablation Test}

We validate our VVNet design with a set of ablation tests on the SUNCG dataset. 
Proceedings of the Twenty-Seventh International Joint Conference on Artificial Intelligence (IJCAI-18)

\begin{tabular}{|c|c|c|c|c|c|c|c|c|c|c|c|c|c|c|c|}
\hline & scene & comp & letion & \multicolumn{12}{|c|}{ semantic scene completion } \\
\hline Network & prec. & recal & IoU & ceil. & floor & wall & win. & chair & bed & sofa & table & tvs & furn. & objs. & avg. \\
\hline SSCNet & 76.3 & 95.2 & 73.5 & 96.3 & 84.9 & 56.8 & 28.2 & 21.3 & 56.0 & 52.7 & 33.7 & 10.9 & 44.3 & 25.4 & 46.4 \\
\hline SSCNet* & 0.4 & 89.7 & 82.0 & 97.8 & 88.2 & 59.4 & 37.3 & 39.2 & 77.9 & 68.9 & 48.3 & 31.5 & 56.8 & 44.9 & 59.1 \\
\hline SSCNet*-half & 90.5 & 89.5 & 81.9 & 97.8 & 88.0 & 60.8 & 34.8 & 39.8 & 77.5 & 69.5 & 47.8 & 29.8 & 56.0 & 44.8 & 58.8 \\
\hline VVNet-120-half & 0.7 & 89.6 & 82.1 & 97.9 & 85.2 & 59.4 & 47.5 & 44.2 & 77.4 & 71.1 & 49.3 & 34.2 & 58.2 & 49.0 & 61.3 \\
\hline VVNet-120-depth & 90.6 & 89.6 & 82.0 & 97.6 & 84.8 & 58.6 & 44.5 & 44.8 & 77.6 & 70.7 & 48.8 & 33.2 & 57.8 & 46.2 & 60.4 \\
\hline VVNet-120 & 90.8 & 90.0 & 82.5 & 97.9 & 85.4 & 58.6 & 49.2 & 45.3 & 79.2 & 71.8 & 50.3 & 37.3 & 62.0 & 50.9 & 62.5 \\
\hline VVN & 90.8 & 91.7 & 84.0 & 98.4 & 87.0 & 61.0 & 54.8 & 49.3 & 83.0 & 75.5 & 55.1 & 43.5 & 68.8 & 57.7 & 66.7 \\
\hline VVNetR-60 & 90.6 & 92.5 & 83.7 & 97.6 & 86.7 & 60.2 & 54.4 & 47.2 & 80.7 & 75.0 & 53.8 & 39.4 & 66.9 & 56.1 & 65.3 \\
\hline VVNetR-30 & 88.8 & 90.2 & 81.0 & 98.0 & 86.4 & 55.6 & 54.8 & 41.8 & 78.0 & 72.1 & 48.7 & 31.6 & 63.2 & 51.8 & 62.0 \\
\hline
\end{tabular}

Table 1: Performances of different variant VVNet design on the SUNCG dataset. half refers to the network that takes half-resolution image as input. depth refers to the network that use depth only as input.

\begin{tabular}{l|l|ll|lllllllllllll}
\hline & \multicolumn{1}{|c|}{ scene completion } & \multicolumn{10}{c}{ semantic scene completion } \\
\hline Method & prec. & recall IoU & ceil. & floor & wall & win. & chair & bed & sofa & table & tvs & furn. objs. avg. & ob. \\
[Lin et al., 2013] & 58.5 & 49.9 & 36.4 & 0.0 & 11.7 & 13.3 & 14.1 & 9.4 & 29.0 & 24.0 & 6.0 & 7.0 & 16.2 & 1.1 & 12.0 \\
[Geiger and Wang, 2015] & 65.7 & 58.0 & 44.4 & 10.2 & 62.5 & 19.1 & 5.8 & 8.5 & 40.6 & 27.7 & 7.0 & 6.0 & 22.6 & 5.9 & 19.6 \\
\hline SSCNet & 59.3 & $\mathbf{9 2 . 9}$ & 56.6 & 15.1 & 94.6 & 24.7 & 10.8 & 17.3 & 53.2 & 45.9 & 15.9 & 13.9 & 31.1 & 12.6 & 30.5 \\
SSCNet* & 69.7 & 81.3 & 59.8 & 16.1 & $\mathbf{9 4 . 8}$ & 27.0 & 10.1 & $\mathbf{2 0 . 6}$ & 53.2 & 50.1 & 16.7 & $\mathbf{1 4 . 3}$ & 35.5 & 13.0 & 31.9 \\
VVNet-120 & 68.4 & 83.2 & 60.0 & 19.2 & 94.4 & 27.2 & $\mathbf{1 3 . 8}$ & 19.1 & 54.0 & 49.3 & 17.1 & 11.2 & 35.3 & 12.4 & 32.1 \\
VVNetR-120 & $\mathbf{6 9 . 8}$ & 83.1 & $\mathbf{6 1 . 1}$ & 19.3 & $\mathbf{9 4 . 8}$ & 28.0 & 12.2 & 19.6 & $\mathbf{5 7 . 0}$ & 50.5 & $\mathbf{1 7 . 6}$ & 11.9 & $\mathbf{3 5 . 6}$ & 15.3 & 32.9 \\
VVNetR-60 & 68.3 & 85.1 & 60.9 & $\mathbf{2 1 . 6}$ & 94.5 & $\mathbf{2 8 . 6}$ & 12.9 & 19.7 & $\mathbf{5 6 . 3}$ & $\mathbf{5 1 . 0}$ & 17.2 & 10.4 & 35.2 & $\mathbf{1 5 . 6}$ & $\mathbf{3 3 . 0}$ \\
\hline
\end{tabular}

Table 2: The performances of different scene completion methods on the NYU dataset.

Does Higher Image Resolution Help? We downsample the depth input images to half resolution $(320 \times 240)$ and use them for training a SSCNet*-half model and a VVNet120-half model. As shown in Table. 1, the limited TSDF resolution in SSCNet cannot preserve the geometric details in high resolution input image and thus leads to very similar results for both SSCNet* and SSCNet*-half. Note that the TSDF resolution in SSCNet cannot be increased anymore due to the large memory and computational cost of 3D CNN. On the contrary, our method can fully exploit the high resolution input. Compared to VVNet-120-half, the IOUs of the VVNet-120 for SC and SSC tasks improve $0.4 \%$ and $1.2 \%$ respectively.

Does Multi-channel Input Help? To validate the contribution of the normal map to the VVNet result, we train VVNet120-depth with the depth image only as input. Compared to VVNet-120 that takes both depth and normal as the input, the IOUs of the VVNet-120-depth decreases $0.5 \%$ and $2.1 \%$ for SC and SSC tasks respectively, which demonstrates that the normal input helps VVNet to learn the local geometric features. Note that these extra non-depth features (e.g. normal, RGB, etc.) are difficult to be used in 3D CNN training and inference as discussed in several previous methods [Song et al., 2017; Dai et al., 2017; Guedes et al., 2018].

Does Larger Reception Field Help? Table. 1 compares the performances of VVNet-120 and VVNetR-120. For SC and SSC tasks, our new backbone with larger reception field in VVNetR-120 provides $1.9 \%$ and $5.4 \%$ IOU improvements compared to VVNet-120.

\begin{tabular}{l|c|c|c}
\hline & \multicolumn{2}{|c|}{ training } & inference \\
\hline Network & memory & speed & speed \\
\hline SSCNet & $852 \mathrm{M}$ & $912 \mathrm{~ms}$ & $578 \mathrm{~ms}$ \\
\hline VVNet-120 & $846 \mathrm{M}$ & $386 \mathrm{~ms}$ & $75 \mathrm{~ms}$ \\
VVNetR-120, & $712 \mathrm{M}$ & $375 \mathrm{~ms}$ & $74 \mathrm{~ms}$ \\
VVNetR-60, & $336 \mathrm{M}$ & $194 \mathrm{~ms}$ & $51 \mathrm{~ms}$ \\
VVNetR-30, & $246 \mathrm{M}$ & $156 \mathrm{~ms}$ & $45 \mathrm{~ms}$ \\
\hline
\end{tabular}

Table 3: Memory footprints and computational times of different networks for model training and inference.

Trade-offs between View and Volume Networks We compare the performances of VVNet models with different combinations of 2D view and 3D volume CNNs. As shown in Table. 1, the IOUs of the VVNetR-60 is slightly worse than the IOUs of the VVNetR-120 (0.3\% and $1.4 \%$ decreasing for SC and SSC respectively). However, the VVNetR-60 only requires half of memory footprint and $70 \%$ computational time that the VVNetR-120 needs in the training. For VVNetR30 , we observe a relatively large performance drop from the VVNetR-120. A possible reason is that the low resolution feature volume projected from the $2 \mathrm{D}$ features lose too much detailed 3D context information.

\subsection{Evaluation}

we test the performance of VVNet network for semantic scene completion task on all three datasets and compare our method with other existing approaches.

SUNCG For SUNCG dataset, we compare the IOUs of VVNetR-120, SSCNet, and SSCNet* for both SC and SSC tasks. As shown in Table. 1, the VVNetR-120 achieves the best performance in both SC and SSC tasks. Compared to 


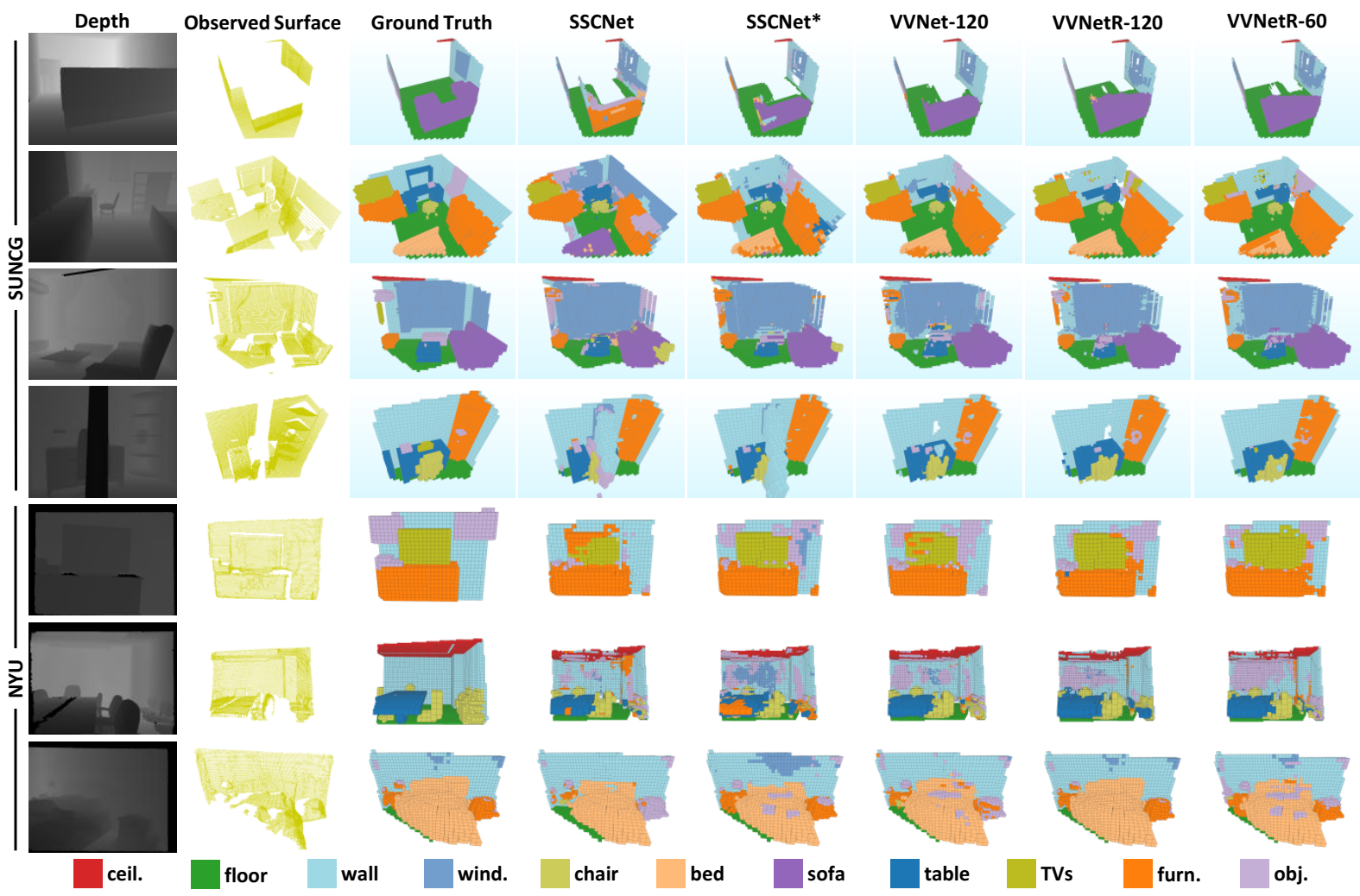

Figure 3: Semantic scene completion results generated by different methods for SUNCG and NYU datasets.

\begin{tabular}{l|ccc}
\hline Method & prec. & recall & IoU \\
\hline [Zheng et al., 2013] & 60.1 & 46.7 & 34.6 \\
[Firman et al., 2016] & 66.5 & 69.7 & 50.8 \\
\hline SSCNet & 75.0 & 96.0 & 73.0 \\
SSCNet $^{*}$ & 83.2 & 92.7 & 78.0 \\
VVNet-120 & 83.3 & 93.1 & 78.5 \\
VVNetR-120 & 86.4 & 92.0 & 80.3 \\
VVNetR-60 & 85.6 & 91.5 & 79.2 \\
\hline
\end{tabular}

Table 4: Performances of different methods on NYUCAD dataset.

SSCNet ${ }^{*}$, the IOUs of VVNetR-120 increase $1.4 \%$ and $5.9 \%$ for SC and SSC tasks respectively. Table. 1 also lists the IOU for each object class. Note that for all object class except floor, the VVNetR-120 achieves the best results. Fig. 3 illustrates the semantic scene completion results generated by different methods.

NYU \& NYUCAD We compare our method with SSCNet* and other existing methods on both NYU and NYUCAD dataset. Table 2 and 4 list the performances of these methods on both datasets. For both NYU and NYUCAD datasets, our VVNet achieves the best performance among all the methods [Lin et al., 2013; Geiger and Wang, 2015]. We believe that the small performance gap between our VVNetR models and SSCNet* on the NYU dataset is caused by the misalignment between the input and output.

Memory Footprint \& Computational Cost Compared to the SSCNet that is based on 3D CNN, our VVNet combines the 2D CNN and 3D CNN and thus significantly reduces both memory footprint and computational cost in training and inference. Table. 3 compares the memory footprints and computational costs of different models for training and inference. Compared to SSCNet, the VVNetR-120 provides much better accuracy and three-times speed up for training, as well as more than 7 times speed up for inference. With $40 \%$ memory footprint of SSCNet in training, the VVNetR-60 offers 4.7 times speed up for training and more than 10 times speed up for inference at the cost of slightly degraded accuracy compared to VVNetR-120. Note that the accuracy of VVNetR-60 is still better than the SSCNet.

\section{Conclusion}

We introduce a view-volume CNN for semantic scene completion from a single depth image. Our method concatenates a $2 \mathrm{D}$ view $\mathrm{CNN}$ and a $3 \mathrm{D}$ volume $\mathrm{CNN}$ with a projection layer and can be trained end-to-end. The VVNet provides a general and flexible framework for fusing 2D and 3D CNNs for efficient 3D learning. We validate our method on both synthetic and real datasets. Results shown that our method significantly improves the result accuracy, reduces the computational cost in training and inference, and offers variant trade-offs between the cost and accuracy.

For the future work, it is interesting to explore the tradeoffs between 2D CNNs and 3D CNNs and find solutions to improve the performance of VVNet with less 3D volume 
layers. Another interesting direction is to extend our viewvolume framework for multiple view $\mathrm{CNN}$ solutions.

\section{References}

[Atapour-Abarghouei and Breckon, 2017] A. Atapour-Abarghouei and T.P. Breckon. Depthcomp: Real-time depth image completion based on prior semantic scene segmentation. In $B M V C$, pages 1-13, 2017.

[Choy et al., 2016] Christopher B Choy, Danfei Xu, JunYoung Gwak, Kevin Chen, and Silvio Savarese. 3d-r2n2: A unified approach for single and multi-view $3 \mathrm{~d}$ object reconstruction. In ECCV, pages 628-644, 2016.

[Dai et al., 2017] Angela Dai, Daniel Ritchie, Martin Bokeloh, Scott Reed, Jürgen Sturm, and Matthias Nießner. Scancomplete: Large-scale scene completion and semantic segmentation for $3 \mathrm{~d}$ scans. arXiv preprint arXiv:1712.10215, 2017.

[Firman et al., 2016] Michael Firman, Oisin Mac Aodha, Simon Julier, and Gabriel J. Brostow. Structured prediction of unobserved voxels from a single depth image. In CVPR, pages 54315440, 2016.

[Geiger and Wang, 2015] Andreas Geiger and Chaohui Wang. Joint $3 \mathrm{~d}$ object and layout inference from a single rgb-d image. In GCPR, pages 183-195, 2015.

[Girdhar et al., 2016] R. Girdhar, D.F. Fouhey, M. Rodriguez, and A. Gupta. Learning a predictable and generative vector representation for objects. In ECCV, pages 484-499, 2016.

[Guedes et al., 2018] Andre Bernardes Soares Guedes, Teofilo Emidio de Campos, and Adrian Hilton. Semantic scene completion combining colour and depth: preliminary experiments. arXiv preprint arXiv:1802.04735, 2018.

[Guo et al., 2015] Ruiqi Guo, Chuhang Zou, and Derek Hoiem. Predicting complete $3 \mathrm{~d}$ models of indoor scenes. arXiv preprint arXiv:1504.02437, 2015.

[Gupta et al., 2013] Saurabh Gupta, Pablo Arbelaez, and Jitendra Malik. Perceptual organization and recognition of indoor scenes from RGB-D images. In CVPR. 2013.

[Gupta et al., 2014] Saurabh Gupta, Ross Girshick, Pablo Arbeláez, and Jitendra Malik. Learning rich features from rgb-d images for object detection and segmentation. In $E C C V$, pages 345-360, 2014.

[Gupta et al., 2015] Saurabh Gupta, Pablo Andrés Arbeláez, Ross B. Girshick, and Jitendra Malik. Aligning 3D models to RGB-D images of cluttered scenes. In CVPR, pages 4731-4740, 2015.

[Häne et al., 2017] Christian Häne, Shubham Tulsiani, and Jitendra Malik. Hierarchical surface prediction for $3 \mathrm{~d}$ object reconstruction. In arXiv preprint arXiv:1704.00710. 2017.

[Ioannidou et al., 2017] Anastasia Ioannidou, Elisavet Chatzilari, Spiros Nikolopoulos, and Ioannis Kompatsiaris. Deep learning advances in computer vision with $3 \mathrm{~d}$ data: A survey. ACM Computing Surveys, 50(2):20:1-20:38, 2017.

[Jimenez Rezende et al., 2016] Danilo Jimenez Rezende, S. M. Ali Eslami, Shakir Mohamed, Peter Battaglia, Max Jaderberg, and Nicolas Heess. Unsupervised learning of $3 \mathrm{~d}$ structure from images. In NIPS, pages 4996-5004. 2016.

[Kalogerakis et al., 2017] Evangelos Kalogerakis, Melinos Averkiou, Subhransu Maji, and Siddhartha Chaudhuri. 3D shape segmentation with projective convolutional networks. In $C V P R$, pages 3779-3788, 2017.
[Lin et al., 2013] Dahua Lin, Sanja Fidler, and Raquel Urtasun. Holistic scene understanding for $3 \mathrm{~d}$ object detection with rgbd cameras. In ICCV, pages 1417-1424, 2013.

[Liu et al., 2017] Fangyu Liu, Shuaipeng Li, Liqiang Zhang, Chenghu Zhou, Rongtian Ye, Yuebin Wang, and Jiwen Lu. 3dcnn-dqn-rnn: A deep reinforcement learning framework for semantic parsing of large-scale $3 \mathrm{~d}$ point clouds. In ICCV, pages 5678-5687, 2017.

[Nguyen et al., 2016] D. T. Nguyen, B. S. Hua, M. K. Tran, Q. H. Pham, and S. K. Yeung. A field model for repairing 3d shapes. In $C V P R$, pages 5676-5684, 2016.

[Qi et al., 2016] Charles Ruizhongtai Qi, Hao Su, Kaichun Mo, and Leonidas J. Guibas. Pointnet: Deep learning on point sets for 3d classification and segmentation. In CVPR, pages 652-660, 2016.

[Qi et al., 2017] Charles Ruizhongtai Qi, Li Yi, Hao Su, and Leonidas J. Guibas. Pointnet++: Deep hierarchical feature learning on point sets in a metric space. In NIPS, pages 5105-5114, 2017.

[Ren and Sudderth, 2016] Zhile Ren and Erik B. Sudderth. Threedimensional object detection and layout prediction using clouds of oriented gradients. In CVPR, pages 1525-1533, 2016.

[Rock et al., 2015] Jason Rock, Tanmay Gupta, Justin Thorsen, JunYoung Gwak, Daeyun Shin, and Derek Hoiem. Completing $3 \mathrm{~d}$ object shape from one depth image. In $C V P R$, pages 2484 2493, 2015.

[Silberman et al., 2012] Nathan Silberman, Derek Hoiem, Pushmeet Kohli, and Rob Fergus. Indoor segmentation and support inference from rgbd images. In ECCV, pages 746-760, 2012.

[Song and Xiao, 2016] Shuran Song and Jianxiong Xiao. Deep Sliding Shapes for amodal 3D object detection in RGB-D images. In $C V P R$, pages 808-816, 2016.

[Song et al., 2017] Shuran Song, Fisher Yu, Andy Zeng, Angel X Chang, Manolis Savva, and Thomas Funkhouser. Semantic scene completion from a single depth image. In $C V P R$, pages 17461754, 2017.

[Tulsiani et al., 2017] Shubham Tulsiani, Tinghui Zhou, Alexei A. Efros, and Jitendra Malik. Multi-view supervision for singleview reconstruction via differentiable ray consistency. In CVPR, pages 2626-2634, 2017.

[Wang et al., 2017] Weiyue Wang, Qiangui Huang, Suya You, Chao Yang, and Ulrich Neumann. Shape inpainting using 3d generative adversarial network and recurrent convolutional networks. In ICCV, pages 2298-2306, 2017.

[Wu et al., 2015] Zhirong Wu, Shuran Song, Aditya Khosla, Linguang Zhang, Xiaoou Tang, and Jianxiong Xiao. 3d shapenets: A deep representation for volumetric shape modeling. In $C V P R$, pages 1912-1920, 2015.

[Yan et al., 2016] X. Yan, J. Yang, E. Yumer, Y. Guo, and H. Lee. Perspective transformer nets: Learning single-view $3 \mathrm{~d}$ object reconstruction without 3d supervision. In NIPS 2016, pages 16961704, 2016.

[Yang et al., 2017] Bo Yang, Hongkai Wen, Sen Wang, Ronald Clark, Andrew Markham, and Niki Trignoi. 3d object reconstruction from a single depth view with adversarial learning. In ICCV, pages 679-688, 2017.

[Zheng et al., 2013] Bo Zheng, Yibiao Zhao, Joey C. Yu, Katsushi Ikeuchi, and Song-Chun Zhu. Beyond point clouds: Scene understanding by reasoning geometry and physics. In $C V P R$, pages 3127-3134, 2013. 